\title{
Confinement in Wendelstein 7-X Limiter Plasmas
}

M. Hirsch ${ }^{1}$, A. Dinklage ${ }^{1}$, A. Alonso ${ }^{2}$, G. Fuchert ${ }^{1}$, S. Bozhenkov $^{1}$, U. Höfel ${ }^{1}$,

T. Andreeva ${ }^{1}$, J. Baldzuhn ${ }^{1}$, M. Beurskens ${ }^{1}$, H.-S. Bosch ${ }^{1}$, C.D. Beidler ${ }^{1}$, C. Biedermann ${ }^{1}$, E. Blanco ${ }^{2}$, R. Brakel ${ }^{1}$, R. Burhenn ${ }^{1}$, B. Buttenschön ${ }^{1}$, A. Cappa ${ }^{2}$, A. Czarnecka ${ }^{3}$, M. Endler ${ }^{1}$, T. Estrada ${ }^{2}$, T. Fornal ${ }^{3}$, J. Geiger ${ }^{1}$, O. Grulke ${ }^{1}$, J.H. Harris ${ }^{4}$, D. Hartmann ${ }^{1}$, M. Jakubowski ${ }^{1}$, T. Klinger ${ }^{1}$, J. Knauer ${ }^{1}$, G. Kocsis ${ }^{5}$, R. König ${ }^{1}$, P. Kornejew ${ }^{1}$, A. Krämer-Flecken ${ }^{6}$, N. Krawczyk $^{3}$, M. Krychowiak ${ }^{1}$, M. Kubkowska ${ }^{3}$, I. Ksiazek ${ }^{7}$, A. Langenberg ${ }^{1}$, H.P. Laqua ${ }^{1}$, S. Lazerson $^{8}$, H. Maaßberg ${ }^{1}$, N. Marushchenko ${ }^{1}$, S. Marsen ${ }^{1}$, V. Moncada ${ }^{9}$, D. Moseev ${ }^{1}$, D. Naujoks ${ }^{1}$, M. Otte ${ }^{1}$, N. Pablant ${ }^{8}$, E. Pasch ${ }^{1}$, F. Pisano ${ }^{10}$, K. Rahbarnia ${ }^{1}$, T. Schröder ${ }^{1}$, T. Stange $^{1}$, L. Stephey ${ }^{11}$, T. Szepesi ${ }^{5}$, T. Sunn Pedersen ${ }^{1}$, H. Trimino Mora ${ }^{1}$, H. Thomsen ${ }^{1}$, H. Tsuchiya $^{12}$, Yu. Turkin ${ }^{1}$, T. Wauters ${ }^{13}$, G. Weir ${ }^{1}$, U. Wenzel ${ }^{1}$, A. Werner ${ }^{1}$, R. Wolf ${ }^{1}$, G.A. Wurden $^{14}$, D. Zhang ${ }^{1}$ and the W7-X Team

${ }^{1}$ Max-Planck-Institut für Plasmaphysik, Greifswald, Germany

${ }^{2}$ CIEMAT, Madrid, Spain

${ }^{3}$ IPPLM, Warsaw, Poland

${ }^{4}$ Oak Ridge National Laboratory, Oak Ridge, TN, USA

${ }^{5}$ Wigner Institute, Budapest, Hungary

${ }^{6}$ Forschungszentrum Jülich, Jülich, Germany

${ }^{7}$ Opole University, Opole, Poland

${ }^{8}$ Princeton Plasma Physics Laboratory, Princeton, NJ, USA

${ }^{9}$ CEA Cadarache, France and THERMADIAG

${ }^{10}$ University of Cagliari, Cagliari, Italy

${ }^{11}$ University of Wisconsin, Madison, WI, USA

${ }^{12}$ National Institute for Fusion Science, Toki, Japan

${ }^{13}$ Laboratory for Plasma Physics, ERM-KMS, Brussels, Belgium, TEC Partner

${ }^{14}$ Los Alamos National Laboratory, Los Alamos, NM, USA

\section{E-mail contact of main author: matthias.hirsch@ipp.mpg.de}

\begin{abstract}
Observations on confinement in the first experimental campaign on the optimized Stellarator Wendelstein 7-X are summarized. In this phase W7-X was equipped with five inboard limiters only and thus the discharge length restricted to avoid local overheating. Stationary plasmas are limited to low densities $<2$ to $3 \cdot 10^{19} \mathrm{~m}^{-3}$. With the available 4.3 MW ECR Heating core $T_{e} \sim 8 \mathrm{keV}, T_{i} \sim 1$ to $2 \mathrm{keV}$ are achieved routinely resulting in energy confinement time $\tau_{\mathrm{E}}$ between $80 \mathrm{~ms}$ to $150 \mathrm{~ms}$. For these conditions the plasmas show characteristics of core electron root confinement with peaked $T_{e}$-profiles and positive $E_{r}$ up to about half of the minor radius. Profiles and plasma currents respond to on- and off-axis heating and co- and counter ECCD respectively.
\end{abstract}

\section{Introduction}

The mission of the superconducting Wendelstein 7-X (W7-X) is to explore the capability of optimized stellarators [1,2] with their inherent option for continuous operation as a route towards fusion energy. W7-X is based on the concept of helical-axis, advanced stellarators (HELIAS) and aims at steady-state operation at reactor-relevant collisionality and plasma $\beta$. The W7-X project is conducted in a staged approach: In the first operational phase physics studies of the HELIAS properties will be performed that include the exploration of a path towards a reactor-relevant, divertor-compatible, high-density regime. This phase will use a 
test divertor unit geometrically identical to the steady-state divertor that is planned for the second operational phase, but inertially cooled to reduce the risk of damage due to local overheating. The maximum absorbed heating power with the inertially cooled divertor is restricted to $80 \mathrm{MJ}$ allowing $10 \mathrm{~s}$ plasma pulses at $8 \mathrm{MW}$ heating. During the second operational phase, the fully cooled steady-state divertor and hardened in-vessel components will allow extension of the plasma duration to quasi steady-state discharges at highperformance conditions (a technical limit is given by the availability of first wall cooling for 30 minutes). Prior to the physics exploration a test operation phase was completed with graphite limiter and an unprotected metal wall. It was dedicated to integrated commissioning and tests of components, diagnostics and device control [2]. During this initial campaign, more than 20 diagnostics were successfully commissioned such that a limited physics program could be conducted under machine condition and diagnostic availability constraints. This paper gives an overview of the energy- and particle confinement in this first limiter phase of the W7-X project.

\section{Expectations on confinement in W7-X}

The magnetic field structure of a HELIAS minimizes the inherent drawbacks of classical nonaxisymmetric confinement, namely a) the unacceptably high collisionless transport in the reactor relevant long mean free path $\operatorname{lmf} p$ regime, b) unacceptable orbit losses of fast (heating) particles and c) the degradation of magnetic flux surfaces at high plasma $\beta$. These goals are simultaneously achieved for finite pressure by enforcing poloidal closure of the contours of the second adiabatic invariant $\mathrm{J}$, which is referred to as quasi-isodynamicity and resembles a minimization of the poloidal variation of $|B|$ [1]. This quasi-isodynamicity reduces the radial component of the grad $B$ drift and the resulting neoclassical particle- and energy transport in the $\operatorname{lmfp}$ regime. Simultaneously it also minimizes the Pfirsch-Schlüter currents and thus the pressure induced Shafranov shift so that the selected optimized field structure is largely independent of plasma $\beta$. Moreover, a remaining degree of freedom allows minimize the bootstrap current with benefits for MHD stability and the prevention of disruptions by balancing the driving terms that result from the helicity of the magnetic field with the "tokamak-like" terms from toroidicity. As the second characteristic element of the HELIAS concept, configurations with intrinsically low magnetic shear have been selected where the profile of the rotational transform can be chosen such that low-order rationals and islands are avoided in the confinement region but may be used as inherent diverting structures at its boundary. This island divertor [3] is rather sensitive to asymmetries of the magnetic field or plasma facing components and therefore relies on configuration control which becomes possible through the minimization of plasma currents in a HELIAS. Some elements of this optimization path have already been explored in the predecessor, Wendelstein 7-AS [4], while others like the influence of the selected quasi-isodynamicity magnetic configuration on turbulent transport as well as engineering aspects of a HELIAS device were not considered as design criteria for $\mathrm{W} 7-\mathrm{X}$.

Due to the lack of a disruptive density limit, the stellarator route to a reactor allows for highdensity operation at comparatively moderate temperatures in accordance with divertor needs. Correspondingly high-density steady-state heating scenarios, such as ECRH with $2^{\text {nd }}$ harmonic ordinary wave polarization (O2-mode) heating, need to be developed. High density scenarios at W7-AS [5] and LHD [6] have shown favorable good particle and energy confinement, and even found reduced impurity confinement that prevented impurity accumulation.

A specific role for confinement in helical magnetic confinement concepts plays the radial electric field $E_{r}$ : In a classical stellarator the transport coefficients in the $\operatorname{lmfp}$ regime in the 
plasma core with the low collisionality $v$ greatly exceed those of a tokamak (for a comprehensive overview see [7]): The trajectories of particles trapped in the helical ripple lead to transport coefficients that increase strongly with decreasing collisionality in the socalled $1 / v$ regime resulting in a temperature dependence $\sim T^{(7 / 2)}$. Reduction of the transport coefficients in this $1 / v$ regime via a reduction of the radial component of the $\operatorname{grad} B$ drift was one basic element of the HELIAS optimization. The radial drift of the transport carrying ripple trapped particles may additionally be reduced by finite electric fields $E_{r}$ : The competition between $E_{r} \times B$ rotation and precession of the particles in the helical ripple - albeit it may lead to resonance effects as well - results in a de-trapping of the particles for large $E_{r} \times B$. In the long mean free path this results in a dependence of the transport coefficients for finite $E_{r}$ that are $\sim \sqrt{v}$ (calculations for a W7-X magnetic configuration are given in [7], Fig. 8.27).

The neoclassical radial electron- and ion-fluxes, $\Gamma_{\mathrm{e}}$ and $\Gamma_{\mathrm{i}}$ both depend explicitly on $E_{r}$. Therefore the ambipolarity condition of the neoclassical radial fluxes, $\Gamma_{\mathrm{e}}=\Gamma_{\mathrm{i}}$, allows for certain solutions (so called "roots") with dedicated values of $E_{r}, \Gamma_{\mathrm{e}}$ and $\Gamma_{\mathrm{i}}$ only. For details and examples of these roots using a HELIAS-like configuration see [4, chapter 3.2]. For $T_{e} \sim$ $T_{i}$ in the so-called ion-root, realized at the plasma edge, the strong outward thermodiffusion of the ions is reduced to the level of the electron fluxes by negative values of $E_{r}$. In contrast for regions with $T_{e} \gg T_{i}$, as it is the case for discharges at low to moderate density that are centrally heated by ECRH where strong $T_{e}$ gradients occur, the associated local electron fluxes $\Gamma_{\mathrm{e}}$ are reduced to the level of the local ion fluxes by strong positive $E_{r}$ constituting the so-called Core Electron Root Confinement [5]. CERC characteristics at low to moderate density ECRH discharges - i.e. peaked $T_{e}$ and flat $T_{i}$ profiles together with a positive $E_{r}$ in the central part plasma of the plasma allow the first comparison of transport and confinement in W7-X with its neoclassical predictions. As the peaked $T_{e}$-profiles provide pronounced pressure gradients deep in the plasma core, they also will be the first candidate to measure the Shafranov shift which according to the optimization of W7-X should be reduced. We note that for later reactor relevant high-density operation these CERC conditions may only be relevant for the transient start-up phase. For steady state core plasma with high-density and $T_{e}$ $\sim T_{i}$ at moderate gradients the neoclassically derived $E_{r}$ will be small and the optimization of core confinement relies on the isodynamic reduction of the neoclassical transport coefficients only.

Turbulent fluxes may as well depend on this neoclassically determined value of $E_{r}$ due to the resulting $E \times B$ shear flows. Therefore, transport and confinement in a stellarator could be influenced by neoclassical fluxes even if anomalous / turbulent fluxes prevail as it is the case at the high collisionalities appearing in the plasma edge region.

\section{Test operation phase: technical boundaries and diagnostics}

During its first Operational Phase (abbreviated as OP1.1, 10 weeks of plasma experiments that began at the end of 2015) W7-X was equipped with five uncooled, inboard graphite limiters $[9,10,11]$. A magnetic configuration with $\mathfrak{t}_{a}>5 / 6$, where the 5/6 island chain had been shifted clearly into the plasma, was selected to avoid diverting island structures at the plasma edge [12]. Only a small magnetic configuration variation was acceptable to minimize the risk of uncontrolled loads away from the limiters. To avoid overheating, the absorbed energy was limited to $4 \mathrm{MJ}$ per experiment program, which restricted the plasma discharge length. Stationary discharge scenarios over $6 \mathrm{~s}$ were achieved with $P_{E C R H}$ lowered to $0.6 \mathrm{MW}$ to remain below this energy limit. For plasma breakdown and heating, a total power of 4.3 MW ECRH was routinely available provided by 6 long-pulse $140 \mathrm{GHz}$ gyrotrons that have 
been commissioned for $30 \mathrm{~min}$ operation in preparation for later steady-state experiments [13].

The device was first operated with preprogrammed Helium- or Hydrogen gas injection without feedback density control. For wall conditioning, He glow discharges were used in periods without main magnetic field operation, i.e. prior to daily operation. With the superconducting coils in operation, ECRH recovery discharges with $\mathrm{He}$ as a working gas allowed a temporary $\mathrm{H}$ depletion of the walls. Sequences of low power ECRH conditioning discharges were used for wall cleaning as well. The global gas balance showed that on average about a factor of 4 more particles were pumped than injected, thus outgassing dominated the fueling throughout OP1.1 [14]. The impurity content - intrinsic $(\mathrm{O}, \mathrm{C}, \mathrm{N})$ and injected impurities (Ar, Ne, N) as well as traces of $\mathrm{F}, \mathrm{Cl}, \mathrm{S}_{2}, \mathrm{Fe}$ and $\mathrm{Cu}$ increased with the discharge time since the last glow discharge until it began to limit plasma duration due to a radiative collapse [15] and new wall conditioning became necessary.

The discharge scenarios had highly reproducible temporal (time-traces) and spatial (profile) characteristics. A representative discharge with $3.8 \mathrm{MW}$ heating power provided by 6 gyrotrons and an overall duration of $1.2 \mathrm{~s}$ is given in Fig. 1. In addition to a Hydrogen prefill a few tens of ms prior to the ECRH start time, the density is ramped up by two successive gas pulses recognizable by steps in the dispersion interferometer signal which displays the line integrated density $\left(n_{e} \cdot d l\right)$ measured along an interferometer laser path length of length $l=1.33$ $\mathrm{m}$ passing the plasma center [16]. The volume averaged density reached at the end of the experiment scenario corresponding to the interferometer signal value of $n_{e} \cdot d l=2.510^{19} \mathrm{~m}^{-2}$ is $\bar{n}_{e, \text { interf }}=1.910^{19} \mathrm{~m}^{-3}$. The diamagnetic energy signal $W_{\text {dia }}$ is compensated for currents induced in the vessel by a set of coils nearby the diamagnetic loop that do not encircle the plasma [17]. Maximum stored energy during the campaign was $W_{\text {dia }} \sim 0.5 \mathrm{MJ}(0.4 \mathrm{MJ}$ for the example of Fig.1) resulting in central plasma $\beta$ values with $\beta(0) \sim 2 \%$ at $B=2.5 \mathrm{~T}$. Due to the steep core pressure gradients VMEC code calculations [36] predict a Shafranov-shift of $\delta=3.4 \mathrm{~cm}$ in the triangular shaped toroidal plane. This, however, has not yet been verified due to a lack of precision in mapping the available diagnostic information. Plasma currents, that were measured by Rogowski coils [18], increase throughout the experiment as both the $L / R$-time and the resistive skin time exceed the discharge length. The $L / R$-time $\tau_{\mathrm{L} / \mathrm{R}}$ dominates the total current evolution (for central $T_{e}=8 \mathrm{keV}$ and $\tau_{\mathrm{L} / \mathrm{R}} \sim \mathrm{T}^{3 / 2}$ one derives $\tau_{\mathrm{L} / \mathrm{R}}>20 \mathrm{~s}$ ), while the resistive skin time which is of the order of $\sim 1 \mathrm{~s}$ is indicative of the redistribution of the plasma shielding currents. The total current - Electron Cyclotron Current Drive, ECCD, plus bootstrap current - reached maximum values up to $\sim 2 \mathrm{kA}$ obtained after $1 \mathrm{~s}$ of co-directed ECCD i.e. in the direction of the bootstrap current. With counter directed ECCD (1.8 MW) the bootstrap current could be suppressed such that the total current became $0.1 \mathrm{kA}$. A dipolar structure is observed as well as it is expected for a Pfirsch-Schlüter current pattern [17].

The electron temperature $T_{e}$ was measured by a 32-channel absolutely calibrated ECE radiometer [19]. $T_{e}$-profiles are derived from the second harmonic, extraordinary wave polarization (X2-mode) Electron Cyclotron Emission spectra. The radial localization is derived from radiation transport code calculations with the TRAVIS code [20] which applies a fully-relativistic model of EC-absorption and -emission. Further spectral components include the well-known relativistically downshifted emission from higher-energy thermal electrons in the plasma center, for which the plasma is optically grey. Electron temperature and electron density profile information is further on obtained from Thomson scattering providing a half-profile of $T_{e}$ and $n_{e}$ with 10 channels at the outboard side extending into the limiter shadow [21]. Raman scattering with Nitrogen gas is used for the density calibration. An imaging X-ray Crystal Spectrometer (XICS) probing line integrated impurity spectra 
provided $T_{e}$ profiles in addition to the only $T_{i}$ profile information via profile inversion - with highest accuracy at mid radii [22]. A comparison of profiles is shown in Fig.2. There is a fair agreement between the average densities derived from Thomson scattering $\bar{n}_{e, T S}$ and interferometry $\bar{n}_{e, \text { interf }}$.

In general, the $T_{e}$-profiles are highly reproducible for the same conditions, statistical errors play only a minor role and systematic errors from the diagnostic dominate. Stationary $T_{e}$ profiles for on-axis ECRH show a hot core surrounded by steep temperature gradients at the half minor radius with maximum values of approximately $8 \mathrm{keV}$ in the center obtained at $P_{E C R H}=4 \mathrm{MW}$. The $T_{i}$ profile is rather flat with $T_{i}<T_{e}$ and a gradient appearing outside r/a 0.5. Central $T_{i}$ is increasing continuously throughout the discharge due to electron-ion coupling and only exceeded $2 \mathrm{keV}$ in the best cases. An example is shown in Fig. 1 where $T_{i}$ reaches $1.8 \mathrm{keV}$ during the heating phase of this particular scenario. A subsequent temporary increase to nearly $2 \mathrm{keV}$ is due to the improved electron-in coupling resulting from decreasing $T_{e}$ after the heating has been terminated. At average densities up to $\bar{n}_{e}=2 \cdot 10^{19} \mathrm{~m}^{-3}$ the $T_{e}$ profiles remain almost stationary until heating termination, as shown in the example of Fig. 1. For higher densities, the discharge could not be maintained under stationary conditions with the available heating power.

\section{Observations on confinement in the limiter plasmas}

\subsection{Power balance and global energy confinement}

In the global power balance, $P_{E C R H}-P_{\text {rad }}-P_{C X}-P_{\text {lim }}=d W / d t$, the various components are [23,24]:

$P_{E C R H}$ - the heating power is measured with an accuracy of $\sim 5 \%$ in the transmission line. The absorption of the microwave beam in the plasma launched in second harmonic X-mode is nearly $99 \%$ and measured by diodes on the inner wall of the device [13].

$P_{\text {rad }}$ - the radiative power loss derived from the two bolometer cameras that were available during OP1.1, is typically $P_{r a d} / P_{E C R H}=25$ to $35 \%$ for stationary conditions (assuming toroidal symmetry). Tomography indicates that a radiating belt exists in the outer part of the confinement region. $P_{\underline{r a d}}$ increases towards a radiative collapse, which itself depends on wall conditions. Generally, the radiated power fraction increases with integrated discharge time since the last glow discharge, which is indicative of increasing wall pollution [25].

$P_{\text {lim }}$ - the total power load to the limiters, is estimated from two IR camera systems [26,27], taking into account power load asymmetries that have been derived from thermocouple observations. For stationary conditions at low ECRH power $\left(\mathrm{P}_{\mathrm{ECRH}}=0.6 \mathrm{MW}\right) \sim 60 \%$ of the power is deposit on the limiters. This value drops to values around $40 \%$ for $4 \mathrm{MW}$ of ECRH power.

$P_{C X}$ - the power carried by CX losses resulting from neutral penetration into the confinement region could not be measured during OP1.1.

Finally, in the power balance $\sim 10 \%$ of the $P_{E C R H}$ remains unaccounted for $P_{E C R H}<1 \mathrm{MW}$, and this fraction of missing power increases with heating power [24]. This missing power may be due to the unknown contribution from $C X$-fluxes, $P_{C X}$, expected due to deep fuelling from the limiters. The limiters are located at the inboard side in a toroidal plane with large elongation, which results in a rather small geometric distance to the plasma axis $(\sim 25 \mathrm{~cm})$ due to local flux compression. This configuration may permit deeper fuelling. Another aspect not yet clearly identified is the effect of possible limiter load asymmetries. 
From the $T_{e}, T_{i}$ and $n_{e}$ profiles the confined kinetic energy is calculated assuming $Z_{e f f}=1$ due to unknown impurity content as $W_{k i n}=(3 / 2) \int\left(n_{e} T_{e}+n_{i} T_{i}\right)(d V / d r) d r$. As a first step, the vacuum field is used for the mapping of the profiles and the calculation of $d V / d r$. In general, the ion energy content derived by this way is approximately $W_{i} \sim 1 / 3 W_{e}$. The resulting $W_{k i n}$ typically exceeds the values from the diamagnetic energy $W_{d i a}$ by $25 \%$, which is presumably due to a combined effect of $Z_{\text {eff }}>1$ and imprecise diagnostic mapping [23]. Energy confinement times $\tau_{\mathrm{E}}=W_{k i n} / P_{\mathrm{ECRH}}$ are derived from $W_{k i n}$ as during OP1.1 a larger set of data are available than for $W_{d i a}$ and subsequently reduced by this systematic factor of $25 \%$. For heating power $P_{\mathrm{ECRH}}>1 \mathrm{MW}$ the values of $\tau_{\mathrm{E}}$ scatter around the expectations from ISS04 scaling [28] despite the poor wall conditions. Scenarios with further reduced heating power display a degradation of the global confinement due to increasing radiative losses up to a radiative collapse and have been excluded from the database. From the limited data set available, the energy confinement time scales like $\tau_{\mathrm{E}^{\sim}} P^{\alpha} \cdot n^{\beta}$ where $\alpha=-0.75$ indicating power degradation. A density dependence (nominally $\beta=0.84$ ) can be inferred, but only with large uncertainties due to the small density variation. Finally, absolute values of $\tau_{\mathrm{E}}$ between $80 \mathrm{~ms}$ to $150 \mathrm{~ms}$ are obtained. They correspond to $\tau_{\mathrm{E}} / \tau_{\mathrm{E}, \mathrm{ISS} 04} \sim 0.8$ to 1.4 if compared with the ISS04 scaling indicating a configuration factor $\sim 1.0$. In the ISS04 scaling this configuration factor had been included to account for the variation of confinement in different Stellarator/Heliotron configurations, and it only reaches a value of 1.0 for the configuration with the longest confinement time measured so far (W7-AS, configuration with edge rotational $\left.\mathfrak{t}_{\mathrm{a}}=0.3\right)$ [28].

\subsection{Particle and impurity confinement}

The OP1.1 discharges suffered from a lack of density control and impurity influx. With Helium pulses into Hydrogen discharges and observing the decay of He II lines with a filterscope, only the effective particle confinement times $\tau_{\mathrm{p}}{ }^{*}=\tau_{\mathrm{p}}(1-R)$ can be derived as the He recycling coefficients $R$ are unknown. The measured effective decay time, $\tau_{\mathrm{p}}{ }^{*}$, varies with values of 2 to $3 \mathrm{~s}$ depending on the magnetic configuration. Shifting the 5/6 rational/islands inwards, i.e. away from the fuelling zone, increases the effective He decay time. The same result holds for the fueling efficiency determined by using the slope of the interferometer signal as a proxy [30]. This first result is consistent with a significant impact of islands in the plasma edge on particle balance and $\mathrm{He}$ (impurity) exhaust.

The first (upper) estimate of the impurity confinement time, $\tau_{\text {Imp }}$, is derived from the decay of the Ar impurity concentration in $\mathrm{H}$ discharges with Ar-prefill. This is achieved by using data sufficiently late that ionization is completed and the temporal behavior only reflects the confinement of Ar interfering with recycling flux. Values of $\tau^{*}$ of 150 to $300 \mathrm{~ms}$ are found for highly ionized $>\mathrm{Ar}^{14+}$ near the center and times much greater than $1 \mathrm{~s}$ for ionization states at the edge [22, 31].

\subsection{Local transport analysis and comparison with neoclassical expectations}

Energy- $\left(Q_{e, i}\right)$ and particle fluxes $\left(\Gamma_{e, i}\right)$ integrated over the flux surfaces were derived from fits to the plasma temperature and density profiles (see Fig. 3 for an example). From the ambipolarity condition, $\Gamma_{e}=\Gamma_{i}$, ion root conditions, with negative $E_{r}$ are derived for the outer part of the confinement region in agreement with $E_{r}$ measurements [32]. The peaked $T_{e}$ profile together with a flat $T_{i}$ profile, where $T_{i}(0)<<T_{e}(0)$ in the inner part of the confinement region indicates conditions with Central Electron Root Confinement (CERC). A positive radial electric field (up to $E_{r} \sim+100 \mathrm{~V} / \mathrm{cm}$ ) is calculated from these profiles for central radii up 
to about $r / a=0.5$, which strongly reduces the electron heat flux down to the $\sqrt{v}$ regime level. Indeed, the steep $T_{e}$ gradients cannot be explained for vanishing $E_{r}$, which resembles a $1 / \mathrm{v}$ regime, because if $E_{r}=0$ these gradients would result in electron heat fluxes $\left(Q_{e}+\Gamma_{e} T_{e}\right)$ that exceed the applied heating power (by up to a factor of 6). The CERC conditions are confirmed from the first $E_{r}$ measurements through correlation reflectometry [32] and from the Doppler shift of $\mathrm{Ar}$ lines measured through X-ray imaging spectroscopy [31]. Both measurements show a change in the sign of the radial electric field $E_{r}(r)$ from negative values at the edge (corresponding to the ion root) to positive values $(<100 \mathrm{~V} / \mathrm{m})$ in the core (corresponding to the electron-root) with a boundary at $0.4<r / a<0.6$ that shifts outward with higher heating power [33].

The existence of a $\sqrt{v}$ regime with dominant $E_{r}$ is confirmed from a configuration scan where the effective helical ripple, $\varepsilon_{\text {eff }}$, which is used as a course measure for the remaining (unoptimized) poloidal variation of $|B|$, was varied from $0.7 \%$ to $1.4 \%$. Contrary to the expectations from the $1 / \mathrm{v}$ regime, where the diffusion coefficient scales strongly with $\varepsilon_{\text {eff, }}$ as $D_{1 / N} \sim \varepsilon_{e f f}^{3 / 2} \cdot n^{-1} \cdot T^{7 / 2}$, there are no significant $T_{e^{-}}$-profile changes in the region of strong $T_{e^{-}}$ gradients. This is consistent with the expectation for the $\sqrt{v}$-regime with finite $E_{r}$, where the dependency on $\varepsilon_{e f f}$ vanishes and $D \sqrt{ }_{v} \sim n^{1 / 2} T^{5 / 4} \mathrm{Er}^{-3 / 2}$ [33].

The integral electron and ion heat fluxes derived from the neoclassical calculations are plotted on the right side of Fig.3. The ECRH power launched in X-mode polarization is nearly fully absorbed in the plasma core by electron cyclotron absorption diagnostics as measured from electron cyclotron absorption measurements [13]. Thus under steady state conditions at radii further outward from the heating zone the integrated heat flux which includes all possible transport channels must be given by $P_{E C R H}$. The right plot of Fig.3 shows that - as expected in the outer part of the confinement region with lower $T_{e}$ the neoclassical fluxes are much to low indicating additional fluxes carried by plasma turbulence, radiation or CX neutrals. Moreover, for the conditions in OP1.1 the calculated neoclassical outward fluxes do not reach $P_{E C R H}$ even in the more central region of strong $T_{e}$ gradients as shown for example in the right plot of Fig.3, where the neoclassical fluxes are at most $\sim 1.1 \mathrm{MW}$ for $P_{E C R H}=2.0 \mathrm{MW}$ of launched power. Power step experiments varying $0.6 \mathrm{MW}<P_{E C R H}<4 \mathrm{MW}$ [33] show that this gap between the maximum total neoclassical heat flux (right figure, black line) and the heating power deposited in the plasma core (marked in yellow) increases with $\mathrm{P}_{\mathrm{ECRH}}$ in parallel with the finding of power degradation. This finding is not in contradiction to the observation that the CERC region grows with heating power, since the outer region of the plasma contains the main fraction (typically $>70 \%$ ) of the total confined energy.

\subsection{First observations on transport beyond neoclassics}

Stationary and dynamic transport studies were conducted together with the flexible ECRH system with its option for on- and off-axis heating, power steps and power modulation [13,34]. Although $T_{e}$ and $T_{i}$ profiles are highly reproducible for the same heating and fueling conditions, a thorough transport analysis is hampered by the uncontrolled density and a lack of information on $\mathrm{Z}_{\mathrm{eff}}$.

-> In a small configuration scan shifting the 5/6 rational (islands) radially inward the $T_{e^{-}}$ profiles did not show an influence of this island location on local heat transport.

-> With off-axis heating the strong central peak of the $T_{e}$ profiles vanishes whereas the $T_{e}$ gradients outside the deposition zone remain the same as expected for a local transport model with no indication for profile stiffness (Fig.4). In some cases, with off-axis ECRH, crash events are observed in the ECE spectra with inversion radius near the half minor radius [19]. 
-> Heatwave propagation experiments, with on- and off-axis power modulation of the gyrotrons at various modulation frequency and -depth, allow for the determination of the ECRH power deposition profile as well as for dynamic electron transport studies [34, 35].

-> The plasmas display a variety of dynamic phenomena such as nearly coherent activity and individual transport events, where only the latter events are considered as candidates to affect confinement [19]. In contrast the nearly coherent activity with frequencies around 7 to $10 \mathrm{kHz}$ occurs in several plasmas scenarios with no obvious influence on confinement. It is located near $\sim \mathrm{r} / \mathrm{a}=0.5$ according to ECE measurements and may be related to the $5 / 6$ rational surface $[18,19,35]$.

\section{Conclusion}

Preliminary estimates of confinement from the initial operation phase of the optimized stellarator W7-X exceeded expectations despite a lack of density control and impurity influx due to the use of a limiter configuration with unprotected metal walls. Only low to moderate densities up to average density $\bar{n}_{e}=2 \cdot 10^{19} \mathrm{~m}^{-3}$ could therefore be investigated. Despite these boundary conditions energy confinement times on the order of $150 \mathrm{~ms}$ are obtained. Comparison with ISS04 scaling yields a configuration factor of 1.0 which already corresponds to the expectations for the most favorable helical magnetic configurations so far [28]. The inner half of the plasma has characteristics indicative of Core Electron Root Confinement (CERC) with peaked $T_{e}$ profiles and high central values $T_{e} \sim 8$ up to $10 \mathrm{keV}$, more flat $T_{i}$ profiles with $T_{i}<2 \mathrm{keV}$ and a positive $E_{r}$ in the core in agreement with neoclassical expectations. However, first estimations show that despite the high temperatures anomalous fluxes prevail for these conditions at all radii. A power degradation of confinement is observed and the $T_{e}$-profile responds to on- and off-axis ECR heating with no indication for profile stiffness.

\section{Acknowledgement}

This work has been carried out within the framework of the EUROfusion Consortium and has received funding from the Euratom research and training programme 2014-2018 under grant agreement No 633053. The views and opinions expressed herein do not necessarily reflect those of the European Commission.

\section{REFERENCES}

[1] NÜHRENBERG, J., Phys. Lett. A 114 (1986), 129,

[2] GRIEGER, G., et al., Phys. Fluids B 4 (1992), 2081.

[3] e.g. FENG, Y., et al., Plasma Phys. Control. Fusion 53 (2011), 024009.

[4] HIRSCH, M., et al., Plasma Phys Control Fusion 50 (2008), 053001.

[5] McCORMICK, K., et al. Phys. Rev. Lett. 89 (2002), 015001.

[6] SAKAMOTO, R., et al., Nucl. Fusion 49 (2009) 085002.

[7] BEIDLER, C. et al.,"Helical Confinement Concepts" in Fusion Physics, Ed. by M. Kikuchi, K. Lackner, Minh Quang Tran, International Atomic Energy Agency, Vienna, ISBN 978-92-0-130410-0

[8] YOKOYAMA, M., et al., Nuclear Fusion 47(9) (2007), 1213.

[9] WOLF, R., et al, this conference, OV3-1, submitted to Nucl. Fusion.

[10] KLINGER, T., Plasma Phys. Control Fusion 59 (2017) 014018.

[11] PEDERSEN, T. S. et al., Nucl. Fusion 55 (2015), 126001.

[12] OTTE, M., et al., Plasma Phys. Control. Fusion 58 (2016), 064003.

[13] MARSEN, S., et al., Proc. $43^{\text {rd }}$ EPS Conf. 4.7 - 8.7.2016, Leuven, Belgium, P4.002. 
[14] WAUTERS, T., et al., Proc. $43^{\text {rd }}$ EPS Conf. 4.7 - 8.7.2016, Leuven, Belgium, P4.047.

[15] BUTTENSCHÖN, B., et al., Proc. $43^{\text {rd }}$ EPS Conf. 4.7 - 8.7.2016, Leuven, Belgium, P4.012.

[16] KNAUER, J., et al.,2016, EPS Leuven, Belgium, P4.017.

[17] RAHBARNIA, K., et al., Proc. $43^{\text {rd }}$ EPS Conf. 4.7 - 8.7.2016, Leuven, Belgium, P4.011.

[18] THOMSEN, H., et al., Proc. $43^{\text {rd }}$ EPS Conf. 4.7 - 8.7.2016, Leuven, Belgium, P4.010.

[19] HIRSCH, M., et al., Proc. $43^{\text {rd }}$ EPS Conf. 4.7 - 8.7.2016, Leuven, Belgium, P4.007.

[20] MARUSHCHENKO, N., et al. 2014 Computer Physics Comm. 185(1) 165-176.

[21] PASCH, E., et al., Proc. $43^{\text {rd }}$ EPS Conf. 4.7 - 8.7.2016, Leuven, Belgium, P4.016.

[22] LANGENBERG, A., et al., Proc. $43^{\text {rd }}$ EPS Conf. 4.7 - 8.7.2016, Leuven, Belgium, P4.014.

[23] BOZHENKOV, S., et al., Proc. $43^{\text {rd }}$ EPS Conf. 4.7 - 8.7.2016, Leuven, Belgium, O2.106.

[24] BOZHENKOV S. et al., this conference EX/P5-8 submitted to Nucl. Fusion.

[25] ZHANG, D. et.al, Proc. $43^{\text {rd }}$ EPS Conf. 4.7 - 8.7.2016, Leuven, Belgium, P4.015.

[26] WURDEN, G. A., et al., Nucl. Fusion. 57 (2017), 056036.

[27] NIEMANN, H., et al., 2016 EPS, P4.0005.

[28] YAMADA, H., et al, Nucl. Fusion. 45 (2005), 1684.

[29] FUCHERT, G., et al., manuscript in preparation.

[30] STEPHEY, L., et al., 2016 APS conference, San Jose, submitted to Phys. of Plasmas., in review.

[31] PABLANT, N., et al., Proc. $43^{\text {rd }}$ EPS Conf. 4.7 - 8.7.2016, Leuven, Belgium, P4.013.

[32] KRÄMER-FLECKEN, A., et al., Nucl. Fusion. 57 (2017), 066023.

[33] DINKLAGE, A., et al., Proc. $43^{\text {rd }}$ EPS Conf. 4.7 - 8.7.2016, Leuven, Belgium, O2.107.

[34] HÖFEL, U., et al., Proc. $43^{\text {rd }}$ EPS Conf. 4.7 - 8.7.2016, Leuven, Belgium, P4.008.

[35] WEIR, G.M., et al., $43^{\text {rd }}$ EPS Conf. 4.7 - 8.7.2016, Leuven, Belgium, P4.009.

[36] HIRSHMAN, S.P., Comput. Phys. Commun. 43 (1986) 143-155.

[37] VAN RIJ, W.I., HIRSHMAN , S.P., Phys. Fluids B 1(3) (1989) 563-569.

[38] BOZHENKOV, S. et al., manuscript in preparation. 


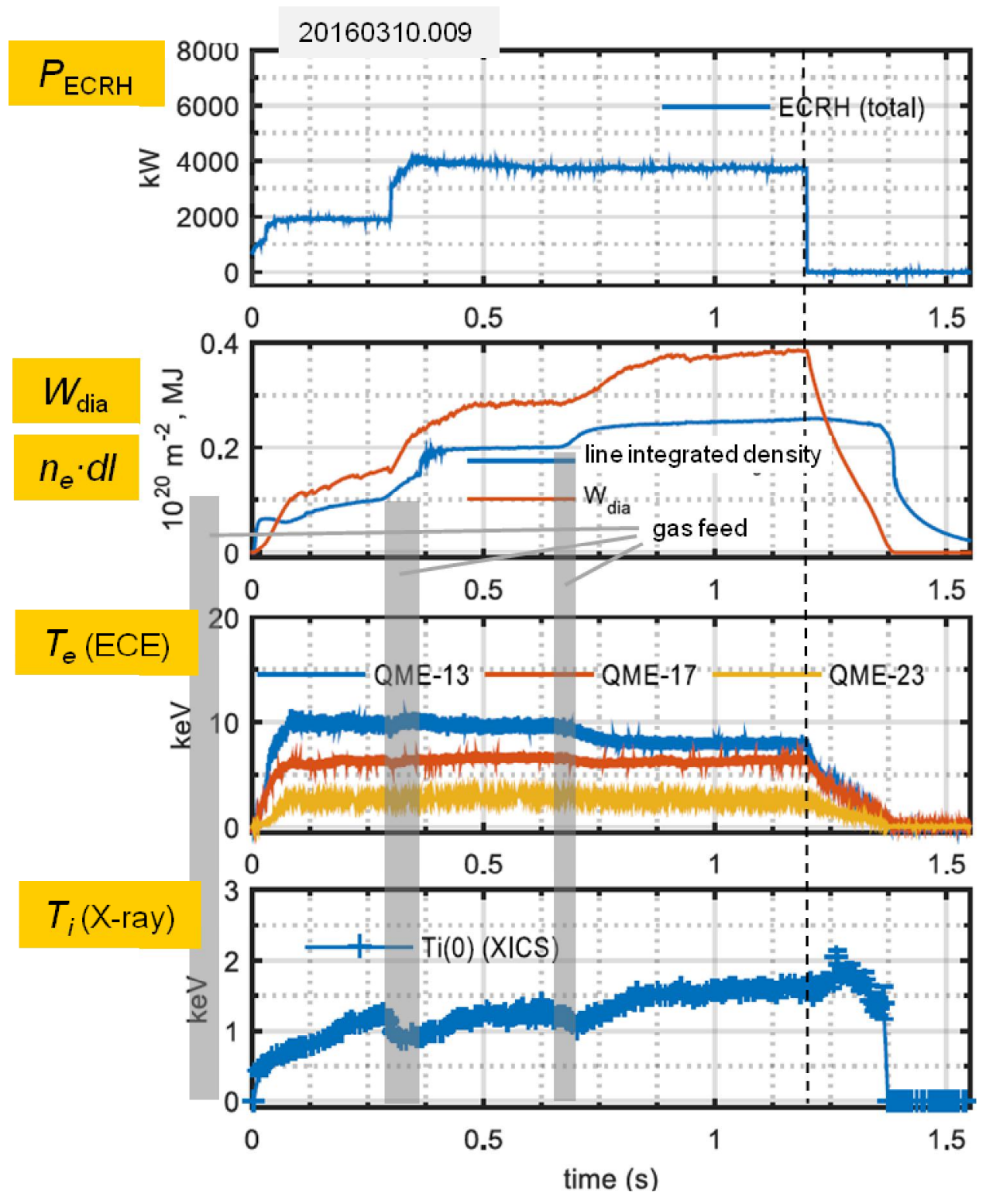

Fig.1: Traces for an ECRH heated W7-X discharge with the origin of the time axis set to the onset of the heating. From top to bottom: Electron Cyclotron Resonance Heating power $\left(\mathrm{P}_{\mathrm{ECRH}}\right)$, diamagnetic energy content $W_{\text {dia }}[17]$ and line integrated density $\left(n_{e} \cdot d l\right)$ measured along an interferometer laser path length of $l=1.33 \mathrm{~m}$ passing the plasma center [16]. The plasma is fuelled by three subsequent hydrogen puffs (timing indicated by grey bars) starting with a prefill already before the heating starts (left). The line integrated density finally reached in the interferometer signal at the end of this particular discharge is $n_{e} \cdot d l=2.510^{19} \mathrm{~m}^{-}$ 2. This corresponds to a volume averaged density of $\bar{n}_{e \text {,int erf }}=1.910^{19} \mathrm{~m}^{-3}$. The two lower frames show the electron temperature $T_{e}$ from selected ECE radiometer channels (probing core, maximum gradient and mid radius) [19] and the central ion temperature $T_{i}$ derived from the X-ray Imaging Crystal Spectrometer (XICS) [22]. 

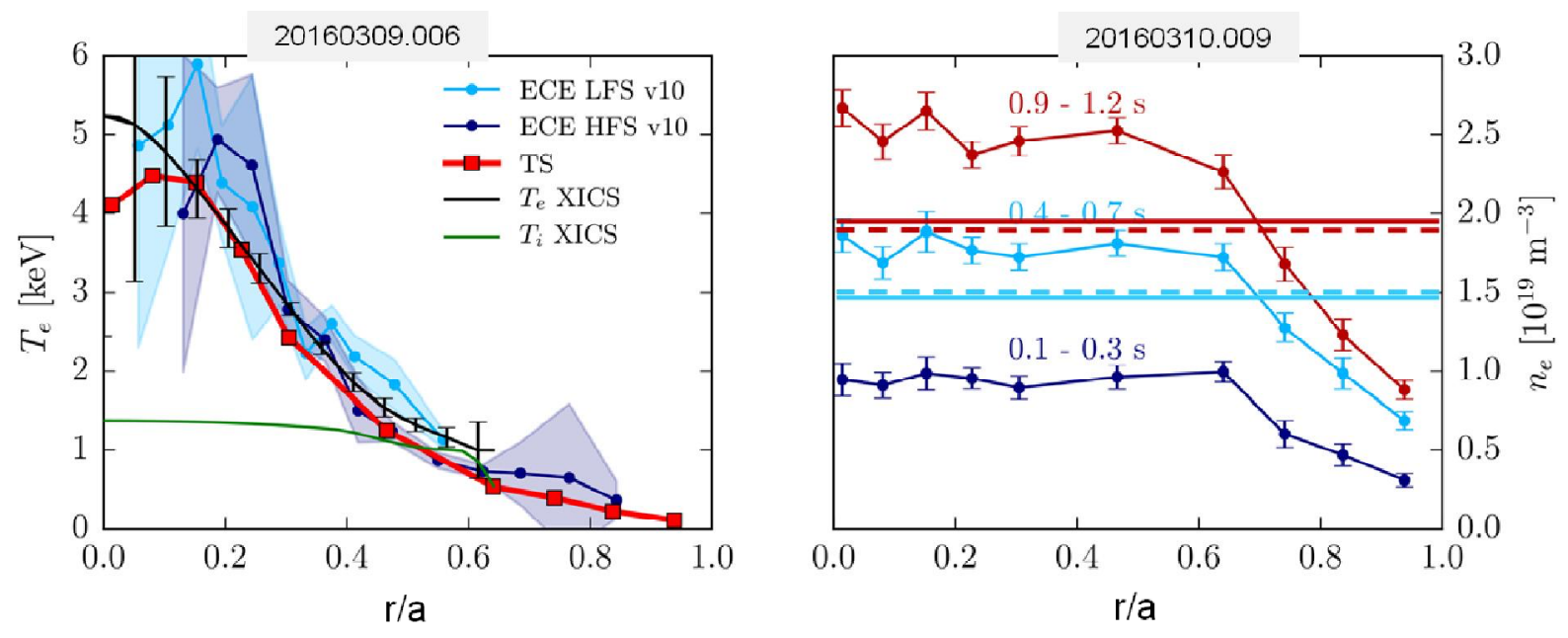

Fig.2: Examples for temperature and density profiles: Left: electron- and ion-temperature measured by ECE, Thomson scattering (TS) and X-ray imaging spectroscopy (XICS) of $\mathrm{Ar}$ impurity lines. Error bars of ECE at low- and high-field side spectra resulting from absolute calibration uncertainties are shaded. Thomson data are plotted without error bars as systematic errors are being investigated; statistical errors are negligible. XICS error bars (black lines) represent inversion uncertainty. A low-beta plasma is shown $\left(\mathrm{P}_{\mathrm{ECRH}}=0.6 \mathrm{MW}\right)$, as the profile comparison bases on a vacuum configuration, the finite pressure Shafranov shift has not yet been included. Right: Electron density profiles measured by Thomson scattering (TS) with the discharge from Fig. 1 taken as an example and the respective time windows given in the figure. The profiles have been taken during the build-up phase of the plasma and from the two subsequent density steps (see Fig.1). For the latter the volume averaged densities derived from these Thomson scattering profiles $\bar{n}_{e, T S}$ are plotted as continuous horizontal lines and compared with the respective volume average densities derived from interferometry $\bar{n}_{e \text {,interf }}$ marked by dashed lines. The limiter position $r / a=1$ corresponds to $r_{\text {eff }}=49 \mathrm{~cm}$. Investigation of systematic errors and mapping of the profile information is ongoing. 

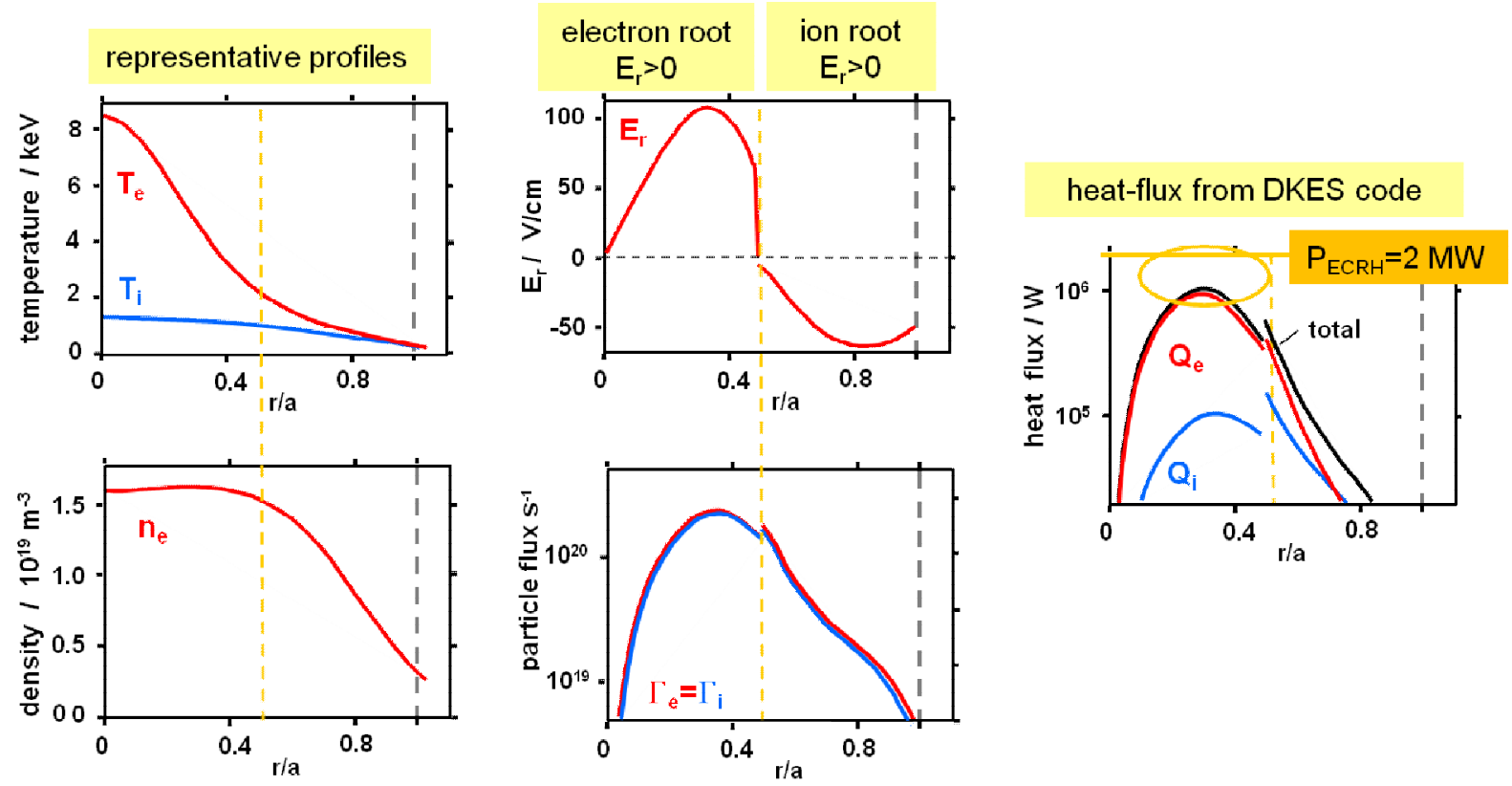

Fig.3: Left column: Temperature and density profiles, middle: derived ambipolar particle fluxes and resulting radial electric field calculated with the DKES code [37] for typical discharge conditions with $2 \mathrm{MW}$ ECRH assuming $Z_{\text {eff }}=1$. On the right side the resulting electron and ion heat fluxes are calculated. The total neoclassical heat flux (right figure, black line) does not at any radius reach the level of the heating power deposit in the center (marked by the yellow line). 

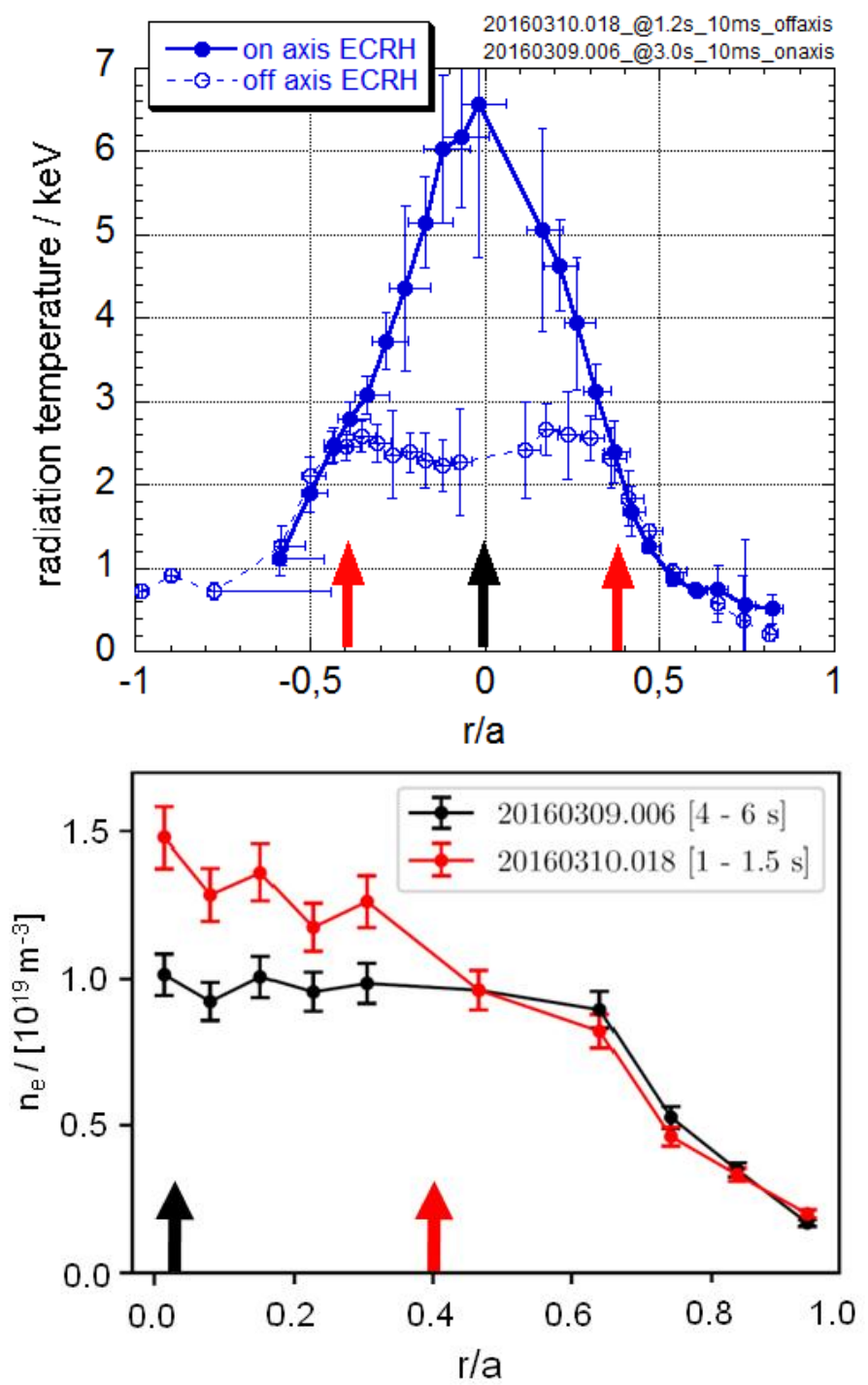

Fig.4: Top: Radiation temperature profiles measured with the ECE radiometer in plasmas heated with low power ECRH (0.6 MW), once on-axis (filled symbols) and second tuned to $20 \mathrm{~cm}$ off-axis (open symbols) the latter corresponding to a deposition radius $\mathrm{r} / \mathrm{a}=0.4$. Heating locations are marked by black (on-axis) and red (off-axis) arrows. Vertical bars indicate systematic errors resulting mainly from the absolute calibration for the individual ECE channels with a hot-cold source, statistical errors from the measurement itself are negligible. Horizontal bars indicate the region from which the microwave emission stems as derived from radiation transport calculations with the TRAVIS code [20] (10\% and 90\% boundary respectively), dots give its center of gravity, resembling the radial localization of the measurements. The good localization of the shown data indicates blackbody conditions and thus that the measured radiation temperature displays the electron temperature. Bottom: Density (half) profiles measured for the same discharges with the available 10 channels of the Thomson scattering system show a tendency to profile peaking with off-axis heating (red symbols). The profiles measured with laser repetition $10 \mathrm{~Hz}$ are averaged over stationary discharge conditions in the time intervals given in the caption. In the error bars of the density measurement systematic errors from the Raman calibration dominate over statistical errors from the measurement [38]. The average density derived from interferometer measurements was $\bar{n}_{e, \text { interf }}=0.810^{19} \mathrm{~m}^{-3}$ in both cases. 